Bull. Korean Math. Soc. 52 (2015), No. 3, pp. 999-1006

http://dx.doi.org/10.4134/BKMS.2015.52.3.999

\title{
POSETS ADMITTING THE LINEARITY OF ISOMETRIES
}

\author{
Jong Youn Hyun, Jeonguin Kim, And Sang-Mok Kim
}

\begin{abstract}
In this paper, we deal with a characterization of the posets with the property that every poset isometry of $\mathbb{F}_{q}^{n}$ fixing the origin is a linear map. We say such a poset to be admitting the linearity of isometries. We show that a poset $P$ admits the linearity of isometries over $\mathbb{F}_{q}^{n}$ if and only if $P$ is a disjoint sum of chains of cardinality 2 or 1 when $q=2$, or $P$ is an anti-chain otherwise.
\end{abstract}

\section{Introduction}

Let $\mathbb{F}_{q}$ be a finite field with $q$ elements, and $\mathbb{F}_{q}^{n}$ the vector space of $n$-tuples over $\mathbb{F}_{q}$. In 1995, Brualdi et al. [1] introduced a non-Hamming metric on $\mathbb{F}_{q}^{n}$ which is associated to an arbitrary poset on $[n]=\{1,2, \ldots, n\}$. It is called a poset metric. The poset metric spaces have been extensively studied in $[1,3,4,5,8]$. We briefly introduce basic notions for poset metric on $\mathbb{F}_{q}^{n}$.

Let $P=([n], \leq)$ be a poset on $[n]$ of coordinate positions of vectors on $\mathbb{F}_{q}^{n}$. A subset $I$ of $P$ is called an order ideal (or a down-set) if $x \in I$ and $y \leq x$ imply $y \in I$. For an arbitrary subset $A$ of $P$, we denote by $\langle A\rangle$ the smallest order ideal of $P$ containing $A$. The $P$-weight of a vector $u=\left(u_{1}, \ldots, u_{n}\right) \in \mathbb{F}_{q}^{n}$ is defined as the cardinality

$$
w_{P}(u)=|\langle\operatorname{supp}(u)\rangle|
$$

of the smallest order ideal of $P$ containing $\operatorname{supp}(u)$, where $\operatorname{supp}(u)=\left\{i \mid u_{i} \neq\right.$ $0\}$. For $u, v \in \mathbb{F}_{q}^{n}$, the $P$-distance $d_{P}(u, v)$ between $u$ and $v$ is defined by

$$
d_{P}(u, v)=|\langle\operatorname{supp}(u-v)\rangle| .
$$

It is well known from [1] that $d_{P}$ defines a metric on $\mathbb{F}_{q}^{n}$. If $P$ is an anti-chain, then $d_{P}$ coincides with the Hamming metric. Therefore, we may view that poset metric is a generalization of the Hamming metric. The theory of poset code generally plays with the properties of the poset metric space $\left(\mathbb{F}_{q}^{n}, d_{P}\right)$.

The isometry group of $\left(\mathbb{F}_{q}^{n}, d_{P}\right)$ is defined by

(3) $\operatorname{Iso}_{P}\left(\mathbb{F}_{q}^{n}\right)=\left\{f: \mathbb{F}_{q}^{n} \rightarrow \mathbb{F}_{q}^{n} \mid d_{P}(f(u), f(v))=d_{P}(u, v)\right.$ for all $\left.u, v \in \mathbb{F}_{q}^{n}\right\}$.

Received June 26, 2014; Revised October 31, 2014.

2010 Mathematics Subject Classification. 94B05.

Key words and phrases. P-isometries, $P$-isometry group, poset metric. 
An element of the isometry group of $\left(\mathbb{F}_{q}^{n}, d_{P}\right)$ is called a $P$-isometry of $\mathbb{F}_{q}^{n}$. It is easy to see that if $f$ is a $P$-isometry of $\mathbb{F}_{q}^{n}$, then it is a bijective map. Moreover, it is easily seen that a linear transformation $f$ of $\mathbb{F}_{q}^{n}$ into itself preserves $P$-distance if and only if it preserves the $P$-weight. Thus we define the automorphism group of $\left(\mathbb{F}_{q}^{n}, d_{P}\right)$, as follows:

(4) $\operatorname{Aut}_{P}\left(\mathbb{F}_{q}^{n}\right)=\left\{f: \mathbb{F}_{q}^{n} \rightarrow \mathbb{F}_{q}^{n} \mid f\right.$ is linear and $w_{P}(f(u))=w_{P}(u)$ for all $\left.u \in \mathbb{F}_{q}^{n}\right\}$.

An element of the automorphism group of $\left(\mathbb{F}_{q}^{n}, d_{P}\right)$ is called a $P$-automorphism of $\mathbb{F}_{q}^{n}$. It is obvious that $\operatorname{Aut}_{P}\left(\mathbb{F}_{q}^{n}\right)$ is a subgroup of $\operatorname{Iso}_{P}\left(\mathbb{F}_{q}^{n}\right)$.

In [8], Panek et al. determine the structure of such automorphism group $\operatorname{Aut}_{P}\left(\mathbb{F}_{q}^{n}\right)$, as follows:

$$
\operatorname{Aut}_{P}\left(\mathbb{F}_{q}^{n}\right) \simeq G(P) \rtimes \operatorname{Aut}(P),
$$

where, if $M_{n \times n}\left(\mathbb{F}_{q}\right)$ denotes the set of all $n \times n$ matrices over $\mathbb{F}_{q}$, then

$$
G(P)=\left\{\left(a_{i j}\right) \in M_{n \times n}\left(\mathbb{F}_{q}\right) \mid \begin{array}{cccc} 
& \mathbb{F}_{q} & \text { if } & i<_{P} j \\
a_{i j} \in & \mathbb{F}_{q}^{*} & \text { if } & i=j \\
& \{0\} & & \text { otherwise }
\end{array}\right\},
$$

and $\operatorname{Aut}(P)$ is the set of all order-preserving-bijections on $P$, i.e., $f \in \operatorname{Aut}(P)$ is a bijection on $P$, provided that if $x \leq y$, then $f(x) \leq f(y)$ for $x$ and $y$ in $P$. In contrast to the early settled result on $\operatorname{Aut}_{P}\left(\mathbb{F}_{q}^{n}\right)$, the generalized question on the structure of $\operatorname{Iso}_{P}\left(\mathbb{F}_{q}^{n}\right)$ is known to be very difficult, as described in $[3,7]$.

Now, we define $\operatorname{Iso}_{P}^{0}\left(\mathbb{F}_{q}^{n}\right)$ as the set of $P$-isometries of $\mathbb{F}_{q}^{n}$ fixing the zero vector $\mathbf{0}$, i.e.,

$$
\operatorname{Iso}_{P}^{0}\left(\mathbb{F}_{q}^{n}\right)=\left\{f: \mathbb{F}_{q}^{n} \rightarrow \mathbb{F}_{q}^{n} \mid f \text { is a } P \text {-isometry and } f(\mathbf{0})=\mathbf{0}\right\} \text {. }
$$

Note that $\operatorname{Aut}_{P}\left(\mathbb{F}_{q}^{n}\right)$ is a $\operatorname{subgroup~of~} \operatorname{Iso}_{P}^{0}\left(\mathbb{F}_{q}^{n}\right)$, and it follows from $[3,8]$ that $\operatorname{Aut}_{P}\left(\mathbb{F}_{q}^{n}\right)=\operatorname{Iso}_{P}^{0}\left(\mathbb{F}_{q}^{n}\right)$ if $P$ is an anti-chain. Conversely, it is worth studying to determine the posets $P$ which satisfy the property that $\operatorname{Aut}_{P}\left(\mathbb{F}_{q}^{n}\right)=\operatorname{Iso}_{P}^{0}\left(\mathbb{F}_{q}^{n}\right)$. We now say that a poset $P$ on $[n]$ admits the linearity of isometries over $\mathbb{F}_{q}^{n}$ if $\operatorname{Aut}_{P}\left(\mathbb{F}_{q}^{n}\right)=\operatorname{Iso}_{P}^{0}\left(\mathbb{F}_{q}^{n}\right)$. In this paper, we characterize the posets on $[n]$ which admit the linearity of isometries over $\mathbb{F}_{q}^{n}$.

Before we state our main result, we give some terminologies in poset theory, as follows. For two disjoint poset $P=(X, \leq)$ and $Q=(Y, \leq)$, the disjoint sum $P+Q$ of $P$ and $Q$ denotes the ordered set on $X \bigcup Y$ such that $x \leq y$ if and only if either $x \leq y$ in $P$ or $x \leq y$ in $Q$. The linear sum $P \oplus Q$ of $P$ and $Q$ is obtained from $P+Q$ by adding the new order relations $x \leq y$ for all $x \in X$ and $y \in Y$. We now state our main result.

Theorem 1.1. Let $P$ be a poset on $[n]$. Then a poset $P$ admits the linearity of isometries over $\mathbb{F}_{q}^{n}$ if and only if $P$ is a disjoint sum of chains of cardinality 2 or 1 when $q=2$, or $P$ is an anti-chain otherwise. 


\section{Proof of Theorem 1.1}

In this section we give a complete proof of Theorem 1.1. For convenience to prove, we define the simple notations for chains and anti-chains as follows. $\mathbf{1}$ denotes the poset with one element. The disjoint sum of $n \mathbf{1}$ 's, written as $n \mathbf{1}$, denotes the anti-chain of cardinality $n$. The linear sum of $n \mathbf{1}$ 's, written as $\mathbf{n}$, denotes the chain of cardinality $n$. We use the notation $\left\{e_{1}, e_{2}, \ldots, e_{n}\right\}$ to denote the canonical basis of $\mathbb{F}_{2}^{n}$.

We first begin with giving a proof of the theorem for the binary case by giving four consecutive propositions and their proofs. Among these propositions, we remark that Propositions 2.3, 2.5, and 2.6 can be also obtained from Theorems 3.3 and 3.9 in [3], through the long and complicate process. However, we now give more direct and simpler proofs, instead.

Proposition 2.1. If $P$ contains 3 as a subposet, then $\operatorname{Aut}_{P}\left(\mathbb{F}_{2}^{n}\right)$ is a proper subgroup of $\operatorname{Iso}_{P}^{0}\left(\mathbb{F}_{2}^{n}\right)$.

Proof. We proceed by induction on $n$. Let $P$ be a poset of cardinality $n$ which contains $\mathbf{3}$ as a subposet. Recall that $\mathbf{3}$ is the poset on [3] $=\{1,2,3\}$ with order relation $1<2<3$. We take $P_{3}$ to be 3 and define a function $f_{3}$ of $\mathbb{F}_{2}^{3}$ into $\mathbb{F}_{2}^{3}$ by $f_{3}(\mathbf{0})=\mathbf{0}, f_{3}\left(e_{1}\right)=e_{1}, f_{3}\left(e_{2}\right)=e_{1}+e_{2}, f_{3}\left(e_{1}+e_{2}\right)=e_{2}, f_{3}\left(e_{3}\right)=e_{1}+e_{2}+e_{3}$, $f_{3}\left(e_{1}+e_{3}\right)=e_{2}+e_{3}, f_{3}\left(e_{2}+e_{3}\right)=e_{1}+e_{3}, f_{3}\left(e_{1}+e_{2}+e_{3}\right)=e_{3}$. It is easy to check that

(7) $\quad d_{P_{3}}\left(f_{s}(u), f_{3}(v)\right)=\left\langle\operatorname{supp}\left(f_{3}(u)+f_{3}(v)\right)\right\rangle=\langle\operatorname{supp}(u+v)\rangle=d_{P_{3}}(u, v)$

for all $u, v \in \mathbb{F}_{2}^{3}$. Using (7), one has that $f_{3}$ is a $P$-isometry of $\mathbb{F}_{2}^{3}$ which is not a linear map (for example, $f_{3}\left(e_{2}+e_{3}\right)=e_{1}+e_{3} \neq e_{3}=f_{3}\left(e_{2}\right)+f_{3}\left(e_{3}\right)$ ). To avoid confusion, let $\langle A\rangle_{P}$ denote the order ideal of $P$ generated by $A$. Assume that a poset $P_{n}$ on $[n]$ and a function $f_{n}$ of $\mathbb{F}_{2}^{n}$ into $\mathbb{F}_{2}^{n}$ have been constructed for which

$$
\left\langle\operatorname{supp}\left(f_{n}(u)+f_{n}(v)\right)\right\rangle_{P_{n}}=\langle\operatorname{supp}(u+v)\rangle_{P_{n}}
$$

for all $u, v \in \mathbb{F}_{2}^{n}$. We define $P_{n+1}$ as a poset on $[n+1]$ which contains $P_{n}$ as a subposet, and a function $f_{n+1}: \mathbb{F}_{2}^{n+1} \rightarrow \mathbb{F}_{2}^{n+1}$ as follows:

$$
f_{n+1}(u)=\left\{\begin{array}{cl}
f_{n}(u) & \text { if } \operatorname{supp}(u) \subseteq[n], \\
f_{n}\left(u^{\prime}\right)+e_{n+1} & \text { if } u=u^{\prime}+e_{n+1}, \operatorname{supp}\left(u^{\prime}\right) \subseteq[n] .
\end{array}\right.
$$

Note that for subsets $A, B$ of $P_{n}$, we have that

$$
\langle A\rangle_{P_{n+1}}=\langle B\rangle_{P_{n+1}}
$$

if $\langle A\rangle_{P_{n}}=\langle B\rangle_{P_{n}}$. Now we will prove by induction that

$$
\left\langle\operatorname{supp}\left(f_{n+1}(u)+f_{n+1}(v)\right)\right\rangle_{P_{n+1}}=\langle\operatorname{supp}(u+v)\rangle_{P_{n+1}}
$$

for all $u, v \in \mathbb{F}_{2}^{n+1}$. We have three cases for these vectors $u, v$ in $\mathbb{F}_{2}^{n+1}$ : Case 1: $\operatorname{supp}(u), \operatorname{supp}(v) \subseteq[n]$.

Case 2: $\operatorname{supp}(u) \subseteq[n], v=v^{\prime}+e_{n+1}, \operatorname{supp}\left(v^{\prime}\right) \subseteq[n]$. 
Case 3: $u=u^{\prime}+e_{n+1}, \operatorname{supp}(u) \subseteq[n], v=v^{\prime}+e_{n+1}, \operatorname{supp}\left(v^{\prime}\right) \subseteq[n]$.

We only give a proof for Case 2 since the other cases can be treated similarly. One has that

$$
\begin{aligned}
& \left\langle\operatorname{supp}\left(f_{n+1}(u)+f_{n+1}(v)\right)\right\rangle_{P_{n+1}} \\
= & \left\langle\operatorname{supp}\left(f_{n}\left(u^{\prime}\right)+f_{n}\left(v^{\prime}\right)+e_{n+1}\right)\right\rangle_{P_{n+1}} \\
= & \left\langle\operatorname{supp}\left(f_{n}\left(u^{\prime}\right)+f_{n}\left(v^{\prime}\right)\right)\right\rangle_{P_{n+1}} \cup\langle n+1\rangle_{P_{n+1}} \\
= & \left\langle\operatorname{supp}\left(u^{\prime}+v^{\prime}\right)\right\rangle_{P_{n+1}} \cup\langle n+1\rangle_{P_{n+1}}(\text { by }(8),(9)) \\
= & \left\langle\operatorname{supp}\left(u^{\prime}+v^{\prime}+e_{n+1}\right)\right\rangle_{P_{n+1}} \\
= & \langle\operatorname{supp}(u+v)\rangle_{P_{n+1}} .
\end{aligned}
$$

It follows from (10) that $f_{n+1}$ is a $P_{n+1}$-isometry of $\mathbb{F}_{2}^{n+1}$ which is not a linear map (for example, $f_{n+1}\left(e_{2}+e_{3}\right)=f_{3}\left(e_{2}+e_{3}\right) \neq f_{3}\left(e_{2}\right)+f_{3}\left(e_{3}\right)=f_{n+1}\left(e_{2}\right)+$ $\left.f_{n+1}\left(e_{3}\right)\right)$. This proves the proposition.

Let $P$ be a poset on $[n]$. For an order ideal $I$ of $P$, we denote by $\max (I)$ (resp. $\min (I))$ the set of maximal (resp. minimal) elements of $I$. For a subset $A$ of $P$, we define $e_{A}=\sum_{i \in A} e_{i}$. By convention, $e_{\varnothing}=\mathbf{0}$.

We begin with the following simple lemma which follows easily from the definition of the $P$-distance.

Lemma 2.2. Let $A, B$ be subsets of a poset $P$. If $\langle A\rangle \subseteq\langle B\rangle$ and $\max (\langle A\rangle) \cap$ $\max (\langle B\rangle)=\varnothing$, then $d_{P}\left(e_{A}, e_{B}\right)=w_{P}\left(e_{B}\right)$.

From now on, $P$ is a poset which does not contain 3 as a subposet. Hence every subset $A$ of $P$ has a decomposition $A=B \cup C$, where $B=A \cap \max (P)$ and $C=A \cap \min (P)$ so that $e_{A}=e_{B}+e_{C}$.

Proposition 2.3. If $P$ contains $1 \oplus 21$ as a subposet, then $\operatorname{Aut}_{P}\left(\mathbb{F}_{2}^{n}\right)$ is a proper subgroup of $\operatorname{Iso}_{P}^{0}\left(\mathbb{F}_{2}^{n}\right)$.

Proof. Let $A$ be a subset of $P$. As mentioned above, we may write

$$
A=B \cup C \text {, where } B=A \cap \max (P) \text { and } C=A \cap \min (P) .
$$

Define a function $f$ of $\mathbb{F}_{2}^{n}$ into $\mathbb{F}_{2}^{n}$ as follows:

$$
f\left(e_{A}\right)=f\left(e_{B}+e_{C}\right)=f\left(e_{B}\right)+e_{C},
$$

where

$$
f\left(e_{B}\right)=\left\{\begin{array}{cc}
e_{B}+\sum_{k, l \in B, k \neq l} e_{\langle k\rangle \cap\langle l\rangle} & \text { if }|B| \geq 2, \\
e_{B} & \text { otherwise. }
\end{array}\right.
$$

First, we will show that $f$ is a $P$-isometry of $\mathbb{F}_{2}^{n}$. For an arbitrary subset $U$ of $P$, we put

$$
t_{U}=\sum_{k, l \in U, k \neq l} e_{\langle k\rangle \cap\langle l\rangle}
$$


Then

$$
\left\langle\operatorname{supp}\left(t_{U}\right)\right\rangle \subseteq\langle U\rangle \text { and } t_{U}=0 \text { if }|U| \leq 1 .
$$

Let $A^{\prime}$ be a subset of $P$ such that

$$
A^{\prime}=B^{\prime} \cup C^{\prime} \text {, where } B^{\prime}=A^{\prime} \cap \max (P) \text { and } C^{\prime}=A^{\prime} \cap \min (P) .
$$

It follows from the definition of $f$ that

$$
\begin{aligned}
d_{P}\left(f\left(e_{A}\right), f\left(e_{A^{\prime}}\right)\right) & =d_{P}\left(f\left(e_{B}+e_{C}\right), f\left(e_{B^{\prime}}+e_{C^{\prime}}\right)\right) \\
& =d_{P}\left(e_{B}+t_{B}+e_{C}, e_{B^{\prime}}+t_{B^{\prime}}+e_{C^{\prime}}\right) \\
& =d_{P}\left(e_{B} \Delta B^{\prime}+e_{C} \Delta C^{\prime}, t_{B}+t_{B^{\prime}}\right),
\end{aligned}
$$

where $A \triangle B$ denote the symmetric difference of sets $A$ and $B$. Note that $t_{B}+t_{B^{\prime}}$ can be represented by the sum of four parts as follows:

$$
t_{B \backslash B^{\prime}}+t_{B^{\prime} \backslash B}+\sum_{k \in B \backslash B^{\prime}, l \in B \cap B^{\prime}} e_{\langle k\rangle \cap\langle l\rangle}+\sum_{k \in B^{\prime} \backslash B, l \in B \cap B^{\prime}} e_{\langle k\rangle \cap\langle l\rangle} .
$$

Using (11) the supports of each part in the right hand side of (13) are contained in the order ideal generated by $B \triangle B^{\prime}$. By Lemma 2.2, (12) becomes $w_{P}\left(e_{B} \triangle B^{\prime}+e_{C \triangle C^{\prime}}\right)=d_{P}\left(e_{B} \triangle B^{\prime}, e_{C} C_{C^{\prime}}\right)=d_{P}\left(e_{A}, e_{A^{\prime}}\right)$. Next, we will show that $f$ is not a linear map if $P$ contains $\mathbf{1} \oplus 2 \mathbf{1}$. Let $\mathbf{1} \oplus 2 \mathbf{1}=\{j\} \oplus\left\{i_{1}, i_{2}\right\}$. For a contradiction, assume that $f$ is a linear. Then

$$
e_{i_{1}}+e_{i_{2}}=f\left(e_{i_{1}}\right)+f\left(e_{i_{2}}\right)=f\left(e_{i_{1}}+e_{i_{2}}\right)=e_{i_{1}}+e_{i_{2}}+e_{\left\langle i_{1}\right\rangle \cap\left\langle i_{2}\right\rangle} \cdot
$$

So we have $e_{\left\langle i_{1}\right\rangle \cap\left\langle i_{2}\right\rangle}=\varnothing$, i.e., $\left\langle i_{1}\right\rangle \cap\left\langle i_{2}\right\rangle=\varnothing$, a contradiction to $j \in\left\langle i_{1}\right\rangle \cap\left\langle i_{2}\right\rangle$. This proves the proposition.

The following lemma (See [2]) is essentially the same as that an anti-chain admits the linearity of isometries.

Lemma 2.4. Let $f$ be a P-isometry of $\mathbb{F}_{2}^{n}$ which fixes the origin. Let $A$ be a subset of $\min (P)$. Then $f\left(e_{A}\right)=\sum_{i \in A} f\left(e_{i}\right)$.

From now on, we assume that $P$ does not contain both $\mathbf{3}$ and $\mathbf{1} \oplus 2 \mathbf{1}$. Therefore $P$ is the disjoint union of $Q_{s}$ 's and an anti-chain, where $Q_{s}=s \mathbf{1} \oplus \mathbf{1}$ $(s \geq 1)$.

Proposition 2.5. If $P$ contains $2 \mathbf{1} \oplus \mathbf{1}$ as a subposet, then $\operatorname{Aut}_{P}\left(\mathbb{F}_{2}^{n}\right)$ is a proper subgroup of $\operatorname{Iso}_{P}^{0}\left(\mathbb{F}_{2}^{n}\right)$.

Proof. Let $s \geq 2$ and $\sigma$ a nontrivial permutation on $\min \left(Q_{s}\right)$. Let us write $\sigma(u)$ for $\sum_{i \in \operatorname{Supp}(u)} e_{\sigma(i)}$. Define a map $g$ of $\mathbb{F}_{2}^{s+1}$ into $\mathbb{F}_{2}^{s+1}$ by

$$
g(u)=\left\{\begin{array}{cl}
\sigma(u) & \text { if } \operatorname{supp}(u) \subseteq \min \left(Q_{s}\right) \\
u & \text { otherwise }
\end{array}\right.
$$


We claim that $g$ is a $Q_{s}$-isometry which is not a linear map. If $\operatorname{supp}(u)$ and $\operatorname{supp}(v)$ are both subsets of the anti-chain $\min \left(Q_{s}\right)$, then by Lemma 2.4, $\sigma$ can be extended to the $Q_{s}$-isometry of $\mathbb{F}_{2}^{s+1}$ by fixing the maximal element of $Q_{s}$. If neither is a subset of $\min \left(Q_{s}\right)$, then $g(u)=u$ and $g(v)=v$, so $d_{Q_{s}}(g(u), g(v))=d_{Q_{s}}(u, v)$. Thus, to prove that $g$ preserves the $Q_{s}$-distance, it is sufficient to consider vectors $u, v$ in $\mathbb{F}_{2}^{s+1}$ which satisfy $\operatorname{supp}(u) \subseteq \min \left(Q_{s}\right)$ and $\operatorname{supp}(v) \nsubseteq \min \left(Q_{s}\right)$. For such $u$ and $v$ in $\mathbb{F}_{2}^{s+1}$, one obtains $d_{P}(g(u), g(v))=$ $d_{P}(\sigma(u), v)=s+1=d_{P}(u, v)$. It remains to show that $g$ is not a linear map. Let $\max \left(Q_{s}\right)=\{i\}$. Since $\sigma$ is a nontrivial permutation on $\min \left(Q_{s}\right)$, there are distinct elements $j, k$ in $\min \left(Q_{s}\right)$ such that $\sigma(j)=k$. From this, one has $g\left(e_{i}+e_{j}\right)=e_{i}+e_{j} \neq e_{i}+e_{k}=g\left(e_{i}\right)+g\left(e_{j}\right)$. This proves the claim. It follows from the assumption of the proposition that $P=Q_{s} \cup P^{\prime}$ with $s \geq 2$. We may write $u \in \mathbb{F}_{2}^{n}$ as $u=\left(u^{1}, u^{2}\right)$ with $\operatorname{supp}\left(u^{1}\right) \subseteq Q_{s}$ and $\operatorname{supp}\left(u^{2}\right) \subseteq P^{\prime}$. Define $f(u)=f\left(u^{1}, u^{2}\right)=\left(g\left(u^{1}\right), u^{2}\right)$. It is easy to see that $f$ is a $P$-isometry which is not a linear map. This proves the proposition.

Now assume that $P$ contains none of $\mathbf{3}, 2 \mathbf{1} \oplus \mathbf{1}, \mathbf{1} \oplus 2 \mathbf{1}$ as a subposet. Then $P=m \mathbf{1} \stackrel{\circ}{\cup} \mathbf{2}$ for some $m, n \geq 0$.

Proposition 2.6. If $P=m \mathbf{1} \cup n \mathbf{2}$ for some $m, n \geq 0$, then every $P$-isometry of $\mathbb{F}_{2}^{n}$ which fixes the origin is a linear map.

Proof. We will show that

$$
f\left(e_{A}\right)=\sum_{i \in A} f\left(e_{i}\right)
$$

for any subset $A$ of $P$. We proceed by induction on the cardinality of $A$. It is clear that (15) holds for every $A$ with $|A|=1$. Assume that (15) holds for every subset of cardinality less than $s$ and that $|A|=s$. If $A \subseteq \min (P)$, then the result follows from Lemma 2.4. So we may assume that $A \cap \max (P) \neq \varnothing$. Take an element $i \in A \cap \max (P)$. Since $f$ is a bijection of $\mathbb{F}_{2}^{n}$, we may write

$$
f\left(e_{A}\right)=f\left(e_{A \backslash\{i\}}+e_{i}\right)=f\left(e_{A \backslash\{i\}}\right)+f(u)
$$

for some $u \in \mathbb{F}_{2}^{n}$. By induction hypothesis,

$$
f\left(e_{A}\right)=\sum_{i \in A \backslash\{i\}} f\left(e_{i}\right)+f(u) .
$$

We claim that $u=e_{i}$. Since $f$ preserves the $P$-distance, we obtain the following three relations from (15):

$$
\begin{gathered}
|\langle A\rangle|=d_{P}\left(e_{A \backslash\{i\}}, u\right), \\
2=|\langle i\rangle|=|\langle\operatorname{supp}(u)\rangle|, \\
w_{P}\left(e_{A}+u\right)=|\langle A \backslash\{i\}\rangle| .
\end{gathered}
$$


For example, the relation (17) is derived as follows:

$$
\begin{aligned}
|\langle i\rangle|=w_{P}\left(e_{i}\right) & =d_{P}\left(e_{A}, e_{A \backslash\{i\}}\right) \\
& =d_{P}\left(f\left(e_{A}\right), f\left(e_{A \backslash\{i\}}\right)\right) \text { (since } f \text { is a } P \text {-isometry) } \\
& =w_{P}\left(f\left(e_{A}\right)+f\left(e_{A \backslash\{i\}}\right)\right) \\
& =w_{P}(f(u))(\text { by }(15)) \\
& =w_{P}(u)(\text { since } f \text { is a } P \text {-isometry) } \\
& =|\langle\operatorname{supp}(u)\rangle| .
\end{aligned}
$$

Since $|\langle\operatorname{supp}(u)\rangle|=|\langle i\rangle|=2$, we have two possibilities:

(i) $\quad\langle\operatorname{supp}(u)\rangle=\langle k\rangle$ for some $k \in \max (P)$, and

Notice that

$$
\text { (ii) } \operatorname{supp}(u)=\{k, l\} \text { for some } k, l \in \min (P) \text {. }
$$

If (20) happens, by (18), we have $|\langle A \backslash\{i\}\rangle|=w_{P}\left(e_{A}+e_{k}+e_{l}\right) \geq|\langle A\rangle|$, a contradiction to (21). Thus (19) must hold. So we may write $u=e_{k}+a e_{l}$, $a \in \mathbb{F}_{2}$ and $l<k$ in $P$. It follows from (18) that

$$
|\langle A \backslash\{i\}\rangle|=w_{P}\left(e_{A}+e_{k}+a e_{l}\right) .
$$

From (21) and (22) we deduce that $k \in A$. It now follows from (16) that

$$
|\langle A\rangle|=d_{P}\left(e_{A \backslash\{i\}}, e_{k}+a e_{l}\right)=d_{P}\left(e_{(A \backslash\{i\})}+e_{k}, a e_{l}\right) .
$$

From (23) and $k \in A$ we deduce that $i=k$. From $i=k$ and (22) we see that

$$
|\langle A \backslash\{i\}\rangle|=w_{P}\left(e_{A \backslash\{i\}}+a e_{l}\right) \text { and } l<i \text { in } P .
$$

Therefore, $a=0$ and so $u=e_{k}$. This proves the proposition.

Theorem 1.1 follows from the four previous propositions.

Next, we deal with the $q$-ary case for $q>2$. Recall that the height of an element $j$ in a poset $P$ is the maximum of lengths of chains descending from $j$.

Proposition 2.7. Let $P$ be a poset on $[n]$ and let $q>2$. Then $P$ admits the linearity of isometries over $\mathbb{F}_{q}^{n}$ if and only if $P$ is an anti-chain.

Proof. As mentioned in Introduction, if $P$ is an anti-chain, it admits the linearity of isometries over $\mathbb{F}_{q}^{n}$. Conversely, let $P$ be a poset on $[n]$ which contains an element $j$ of height one. Then we can choose a minimal element $i$ in $P$ so that $i<_{P} j$. We may assume that $i=1, j=2$. Define a function $f$ of $\mathbb{F}_{q}^{n}$ into $\mathbb{F}_{q}^{n}$ by $f\left(e_{1}+e_{2}\right)=e_{2}, f\left(e_{2}\right)=e_{1}+e_{2}$ and $f(u)=u$ otherwise. It is easy to check that $f$ is not a linear map. Indeed, $f\left(2 e_{2}\right)=2 e_{2} \neq 2\left(e_{1}+e_{2}\right)=2 f\left(e_{2}\right)$. To show that $f$ is a $P$-isometry of $\mathbb{F}_{q}^{n}$, we just consider the following cases since the other cases are clearly satisfied: For $u \neq e_{2}, e_{2}+e_{1}$ we have $d_{P}\left(e_{1}, e_{2}+u\right)=w_{P}\left(e_{2}+u\right)$ by Lemma 2.2. Therefore, 
we get $d_{P}\left(f\left(e_{2}\right), f(u)\right)=d_{P}\left(e_{2}, u\right)$ and $d_{P}\left(f\left(e_{1}+e_{2}\right), f(u)\right)=d_{P}\left(e_{1}+e_{2}, u\right)$. This proves the proposition.

Acknowledgment. The present research has been conducted by the Research Grant of Kwangwoon University in 2014.

\section{References}

[1] R. A. Brualdi, J. Graves, and K. M. Lawrence, Codes with a poset metric, Discrete Math. 147 (1995), no. 1-3, 57-72.

[2] H. Fripertinger, Enumeration of the semilinear isometry classes of linear codes, Bayreuth. Math. Schr. 74 (2005), 100-122.

[3] J. Y. Hyun, A subgroup of the full poset-isometry group, SIAM J. Discrete Math. 24 (2010), no. 2, 589-599.

[4] J. Y. Hyun and H. K. Kim, Maximum distance separable poset codes, Des. Codes Cryptogr. 48 (2008), no. 3, 247-261.

[5] H. K. Kim and D. S. Krotov, The poset metrics that allow binary codes of codimension $m$ to be $m$-, $(m-1)$-, or $(m-2)$-perfect, IEEE Trans. Inform. Theory 54 (2008), no. 11, $5241-5246$.

[6] F. J. MacWilliams and N. J. Sloane, The Theory of Error-Correcting Codes, NorthHolland, Amsterdam, 1998

[7] L. Panek, M. Firer, and M. M. S. Alves, Symmetry groups of Rosenbloom-Tsfasman spaces, Discrete Math. 309 (2009), no. 4, 763-771.

[8] L. Panek, M. Firer, H. K. Kim, and J. Y. Hyun, Groups of linear isometries on poset structures, Discrete Math. 308 (2008), no. 18, 4116-4123.

JONG YOUN HYUN

Institute of Mathematical Sciences

EWha Womans University

SeOUl 120-750, Korea

E-mail address: hyun33@postech.ac.kr

JEONGJIN KIM

Department of Mathematics

MYUNGJI UNIVERSITY

YONGIN 449-728, KoreA

E-mail address: jjkim@mju.ac.kr

SANG-MOK KIM

Department of MATHEMATICS

KWANGWOON UNIVERSITY

SeOul 139-701, Korea

E-mail address: smkim@kw.ac.kr 